LAWRENCE LIVERMORE NATIONAL LABORATORY

\title{
Coarse-Grained Molecular Dynamics for Computer Modeling of Nanomechanical Systems
}

R. E. Rudd

November 11, 2003

Submitted for publication in International Journal on Multiscale Computational Engineering 


\section{DISCLAIMER}

This document was prepared as an account of work sponsored by an agency of the United States Government. Neither the United States Government nor the University of California nor any of their employees, makes any warranty, express or implied, or assumes any legal liability or responsibility for the accuracy, completeness, or usefulness of any information, apparatus, product, or process disclosed, or represents that its use would not infringe privately owned rights. Reference herein to any specific commercial product, process, or service by trade name, trademark, manufacturer, or otherwise, does not necessarily constitute or imply its endorsement, recommendation, or favoring by the United States Government or the University of California. The views and opinions of authors expressed herein do not necessarily state or reflect those of the United States Government or the University of California, and shall not be used for advertising or product endorsement purposes.

This is a preprint of a paper intended for publication in a journal or proceedings. Since changes may be made before publication, this preprint is made available with the understanding that it will not be cited or reproduced without the permission of the author. 


\title{
Coarse-Grained Molecular Dynamics for Computer Modeling of Nanomechanical Systems
}

\author{
Robert E. Rudd* \\ Lawrence Livermore National Laboratory, Condensed Matter Physics Div., L-045, Livermore, CA 94551 USA
}

(Dated: November 2, 2003)

Unique challenges for computer modeling and simulation arise in the course of the development and design of nanoscale mechanical systems. Materials often exhibit unconventional behavior at the nanoscale that can affect device operation and failure. This uncertainty poses a problem because of the limited experimental characterization at these ultra-small length scales. In this Article we give an overview of how we have used concurrent multiscale modeling techniques to address some of these issues. Of particular interest are the dynamic and temperature-dependent processes found in nanomechanical systems. We focus on the behavior of sub-micron mechanical components of Micro-Electro-Mechanical Systems (MEMS) and Nano-Electro-Mechanical Systems (NEMS), especially flexural-mode resonators. The concurrent multiscale methodology we have developed for NEMS employs an atomistic description of millions of atoms in relatively small but key regions of the system, coupled to, and run concurrently with, a generalized finite element model of the periphery. We describe two such techniques. The more precise model, Coarse-Grained Molecular Dynamics (CGMD), describes the dynamics on a mesh of elements, but the equations of motion are built up from the underlying atomistic physics to ensure a smooth coupling between regions governed by different length scales. In many cases the degrees of smoothness of the coupling provided by CGMD is not necessary. The hybrid Coupling of Length Scales (CLS) methodology, combining molecular dynamics with conventional finite element modeling, provides a suitable technique for these cases at a greatly reduced computation expense. We review these models and some of the results we have obtained regarding size effects in the elasticity and dissipation of nanomechanical systems.

\section{MEMS MODELING THROUGH MULTISCALE SIMULATION}

Micro-Electro-Mechanical Systems (MEMS) are computer chips with moving parts, i.e., mechanical components that act as sensors and actuators. The control circuitry and the mechanical components are on the same semiconductor substrate, both fabricated using lithographic techniques. The mechanical components, like the electronic components, can be microscopic or even nanoscopic. In the latter case they are known as Nano-Electro-Mechanical Systems (NEMS). [1] MEMS have been developed for a broad range of applications, including notably accelerometers, pressure gauges and electro-optical devices. [2, 3] They are also currently being developed for biological detection [4] and homeland security applications such as chem/bio-threat detectors. [5] NEMS applications under development range from devices such as gigahertz resonators for communications applications $[1,6,7]$ and ultra-sensitive scanning probe microscopes $[8,9]$, to devices to explore the esoteric worlds of quantum transport [10] and perhaps quantum information processing $[11,12]$. The former applications are in some sense a natural progression driven by the advantages of miniaturization; the latter are tapping into the dramatically new behavior accessible at the nanoscale.

Whether pushing the limits of miniaturization or searching for revolutionary functionality, the development and the design of MEMS and NEMS pose an array of challenges. Their design and optimization is complicated by the multi-physics nature of these devices. Their operation may rely on phenomena as diverse as electrostatics, solid mechanics and microfluidics, depending on the application. To date, MEMS design has been dominated by a cut-andtry paradigm. A design is envisaged, and following some basic calculations, it is fabricated and tested. It has been widely realized that increased use of computer modeling and simulation could significantly reduce the time and cost involved in the design cycle, leading to an effort by many groups to develop computer-based MEMS design tools. [13] These tools are now becoming available.

There are instances in which the problem of MEMS design is further complicated because some of the relevant physics is not well understood. Certainly many of the MEMS materials issues fall into this category, often because surface phenomena that are negligible for macroscopic systems become important in microscopic systems. For many of the applications envisaged for MEMS, it may be necessary to miniaturize the mechanical components of MEMS to such an extent that the materials behavior becomes anomalous. As the dimensions of MEMS devices are reduced to the point that they are comparable to the characteristic size of the microstructure in the solid, a change in the material behavior may be observed. Elastic constants that are isotropic at long length scales where the polycrystalline 
microstructure is effectively homogenized may become anisotropic as the dimensions are reduced below the grain size. Anomalies have been identified in various aspects of materials behavior including elasticity, plasticity, fracture, fatigue and tribology. Although often the form of these effects is known, the materials characterization techniques needed to assess their size and the contributions from various mechanisms may not be available. Of course, the problem is compounded by the small size of the structures.

The requisite experimental techniques will be developed eventually, but until then one way to shed light on these issues is to use computer modeling. A computational approach offers the promise of guidance based on first-principles theory in the absence of complete experimental data. We are particularly interested in the application of concurrent multiscale modeling, which allows the simulation of materials resolved to the atomic level in one region coupled to a model that is less computationally expensive in the surroundings. [14] The coarse-grained model could be continuum mechanics implemented in a finite element model, or as we explain below, it could be a generalization of finite elements developed especially to provide a smooth, seamless coupling to the atomistics. The model in this case is not based on first principles in the quantum mechanical sense, but it is based on proven and transferable classical interatomic potentials. We have used concurrent multiscale modeling to study dynamic and temperature-dependent processes found in nanomechanical systems coupled to larger scale surroundings. [14-16] The ensuing discussion focuses on the behavior of sub-micron MEMS, especially flexural resonators. The concurrent multiscale methodology we have developed for sub-micron MEMS takes advantage of the heterogeneity of the MEMS components. It employs an atomistic description of small but key regions of the system, consisting of millions of atoms, and a mesh-based model of the periphery. The two are run concurrently to attain a self-consistent model of the entire system. [14, 17] We describe two such concurrent multiscale models in this Article. The more precise model, Coarse-Grained Molecular Dynamics (CGMD) [18-20], builds a generalized finite element formalism from the underlying atomistic physics in order to ensure a smooth coupling between regions governed by different length scales. The exacting smoothness of the interface provided by CGMD incurs a computational cost. In many cases, it is possible to move the interface farther into the periphery and use a less expensive model, such as the hybrid Coupling of Length Scale (CLS) model. Either way, CGMD or CLS, the result is a model that accurately describes the behavior of the mechanical components of MEMS down to the atomic scale.

\section{CONCURRENT MULTISCALE MODELING}

In this Section we describe how concurrent multiscale modeling has been applied to model the behavior of some nanoscale mechanical components in NEMS under development. It should be noted that several different approaches to the concurrent multiscale modeling of solid materials have been developed, each optimized for a particular kind of application. [17, 18, 21-23] The methodology we describe here has been optimized for use to simulation the dynamics of nanomechanical systems, including those at finite temperature. The goal is to model an important region of the system, consisting of thousands of cubic nanometers of material, with atomistic precision; surrounding regions of the system are to be modeled at a more coarse-grained level to capture the relevant part of the dynamics but at a much reduced level of computational expense.

There are basically two approaches to concurrent multiscale modeling. The first is to hybridize existing models designed and optimized for certain length scales, and the second is to start with the most accurate fine-scale model and systematically derive models at larger length scales. We will refer to these two approaches as hybrid concurrent multiscale modeling and effective concurrent multiscale modeling. The term "effective" is borrowed from field theory where it is used to describe models that result from integrating out short wavelength or high energy degrees of freedom. [24] The challenge in the hybrid approach is to devise a method to couple two dissimilar models together. The coupling must be physically sensible, free of pathology and computationally tractable. The challenge in the effective modeling approach is to devise a computational framework that allows the calculation of the energies and forces at coarse length scales from those known at the fine length scales. Again, the model must be computationally tractable and free of pathology. The requirement to be physically sensible is replaced by a requirement of controlled self-consistency; i.e., the model at coarse length scales must be consistent with the long wavelength behavior of the underlying fine-scale model. There is invariably some error in the coarse-scale model, if for no other reason because it actually approximates an ensemble of finite-temperature fine-scale systems, but this error should be controlled. In particular, the error should be free of secular terms.

We describe examples of concurrent multiscale modeling in the following Subsections. First we describe the hybrid model known as Coupling of Length Scales. It hybridizes conventional molecular dynamics (a dynamical atomistic technique) with conventional linear finite element modeling based on continuum mechanics. Then we describe an effective multiscale model known as Coarse-Grained Molecular Dynamics. It is based on conventional molecular 


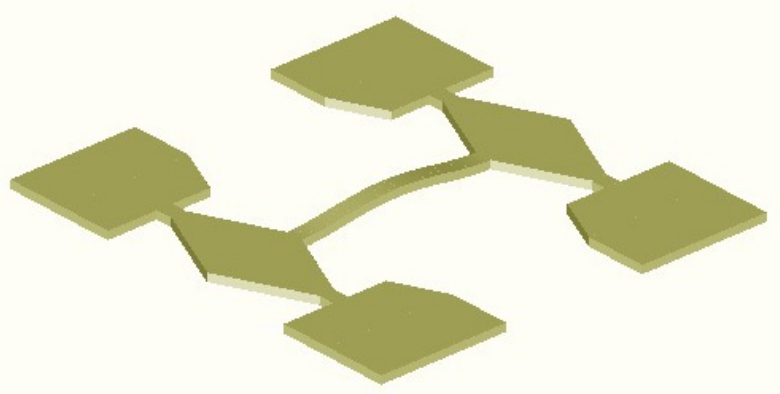

FIG. 1: A three-dimensional model of the sub-micron bridge-type flexural resonator. The central semiconductor bar is attached only at its end points and hence free to oscillate. The bar is deflected in the configuration shown. The oscillations are out of the plane of the substrate. The geometry is modeled on a device fabricated by Prof. Roukes and coworkers. [1]

dynamics. In both cases, the degree of coarse graining is controlled by a mesh, much the way it would be in finite element modeling.

For the discussion of the methodology, we focus on the application to a sub-micron flexural resonator. The geometry of the bridge-type resonator, as simulated, is shown in Fig. 1. This three dimensional geometry is based on a device fabricated by the Roukes group at Caltech. [1] Once the geometry is defined, a mesh is introduced in order to specify which regions are to be coarse grained, as shown in Fig. 2. In particular, the resonating bar and the immediate periphery are modeled using atomistics-the nodes of the mesh and the equilibrium sites of the atoms coincide and are in one-to-one correspondence. Then the mesh size increases moving out farther into the peripheral regions. Here each node of the mesh corresponds to many atoms. It is this reduction in the number of degrees of freedom that increases the efficiency of multiscale simulations. In our simulations the mesh has not been refined on the fly since mesh refinement and coarsening invariably introduces entropy and hence heat. Although the entropy might in principle be limited in such a way as to have a negligible impact on the quantities of interest, we currently do not know how to guarantee or even verify that it would not cause unexpected problems. Thus, we have kept the mesh the same throughout each simulation.

Thus the system is divided into two regions: the molecular dynamics (MD) region described atomistically and the coarse-grained (CG) region described by finite element modeling or a suitable generalization such as CGMD. The region at the interface between $\mathrm{MD}$ and $\mathrm{CG}$ is treated in a special manner in CLS, and we refer to it as the handshaking (HS) region.

\section{CLS: A hybrid atomistic/continuum model}

The CLS methodology is a hybrid combination of molecular dynamics and finite element modeling (FEM). Since the reader may not be familiar with one or both of these modeling approaches, we give a review of some of their salient points. This also provides an opportunity to introduce notation we will use in the discussion of CLS and CGMD. Excellent textbooks are available on MD [25] and FEM [26, 27], so the review given here will be brief.

Molecular dynamics. In MD, a system is modeled at the atomic scale, simulating the motion of the collection of atoms that comprise the system. In nature, atoms interact with each other principally through the quantum mechanical behavior of electrons, which gives rise to metallic, covalent and van der Waals bond formation. In addition individual atoms or clusters may exhibit quasi-static electric or magnetic multipole moments, and thus interact through long-range electrostatic and magnetostatic fields such as in ionic bonding, ferromagnetism and hydrogen bonding. Other more exotic interactions may be present, such as superconductivity and the antiferromagnetism of strongly correlated systems. For our purposes, we will assume that the system is in a normal, weakly correlated state with negligible charge transfer between atoms and no spin polarization (i.e., the electrostatic and magnetostatic forces are essentially zero). Then the interatomic interactions consist of metallic, covalent and/or van der Waals forces. The motion of the ions (atomic nuclei together with their core electrons) is much slower than the motion of the bonding electrons, since the nucleus is so much heavier than the electron. As a result, in virtually all systems the electrons may be assumed to have relaxed to equilibrium at each point of the motion of the ions. This assumption, known as the Born-Oppenheimer approximation, is the basis for removing the electrons from the model and writing effective interatomic potential energies as given functions of the relative positions of the atoms. [28] These potential energies 


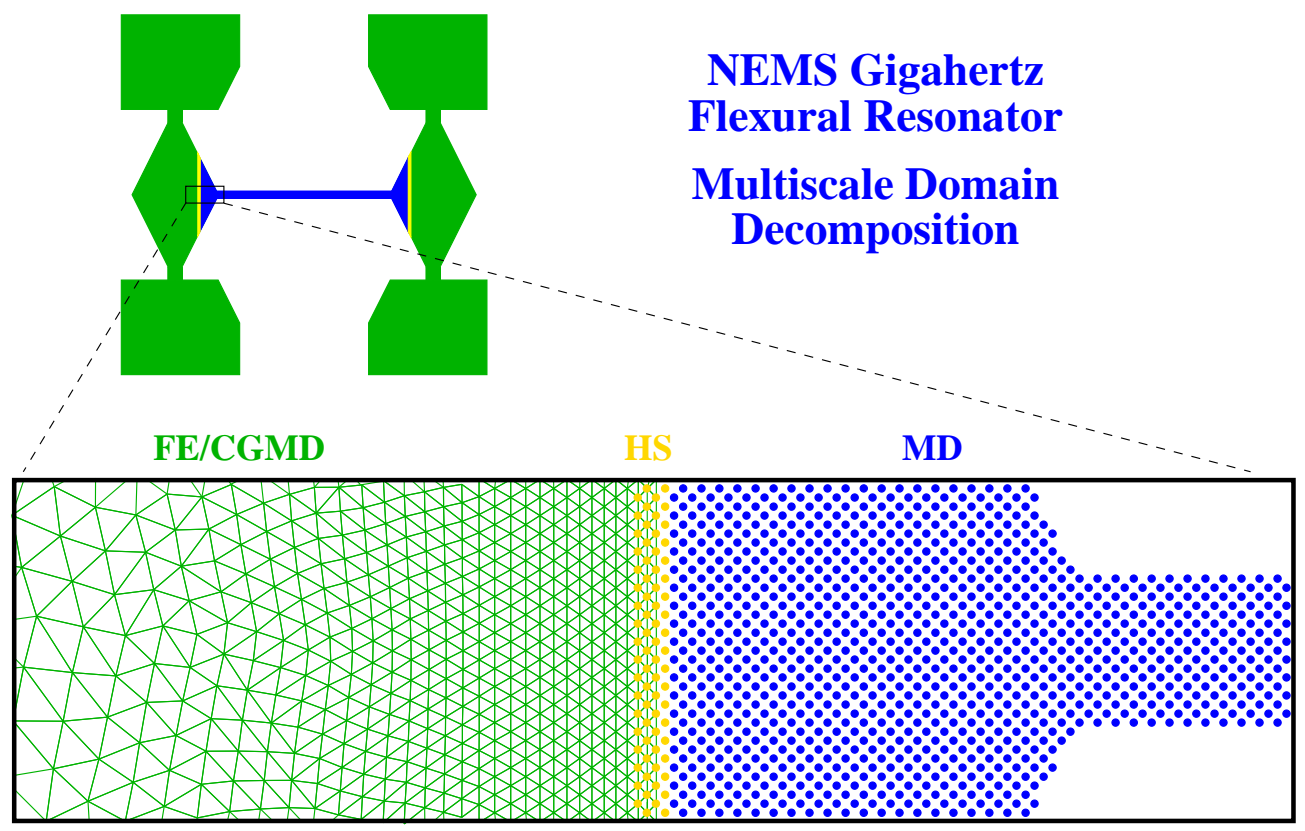

FIG. 2: An example of a multiscale domain decomposition. The sub-micron resonator system is partitioned into molecular dynamics (MD) and finite element (FE) or coarse-grained molecular dynamics (CGMD) regions, as depicted schematically here. [14] The upper diagram shows the entire system, as simulated, with the different domains indicated with different coloring; the lower diagram is an enlarged view of a region at the FE/MD interface. A narrow handshaking region is used to couple the FE and MD regions; in the case of CGMD, the cross-over to atomistics for atomic-sized cells is completely smooth and no handshaking region is required. [18] The simulations are run by displacing the resonator into one of its normal modes and monitoring the ensuing oscillations. No tractions or body force are applied. Periodic boundary conditions are used on the outermost edges of the pads.

may just be functions of the distance between two neighboring atoms. An example of such a pair potential is the well-known Lennard-Jones potential

$$
V\left(r_{\mu \nu}\right)=4 \varepsilon\left(\frac{1}{\left(r_{\mu \nu} / \sigma\right)^{12}}-\frac{1}{\left(r_{\mu \nu} / \sigma\right)^{6}}\right)
$$

where $r_{\mu \nu}$ is the distance between the pair of atoms, $\mu$ and $\nu, \varepsilon$ sets the energy scale and $\sigma$ sets the length scale. [29] The Lennard-Jones potential is suitable for describing the bonding in only a few, special systems such as solid argon. [30] In general, the interatomic potentials contain many-body interactions such as the dependence on coordination (how many atoms surround a given atom) and bond-bending and bond-torsion terms. [31-35] For our simulations of semiconductor NEMS, we have used the Stillinger-Weber silicon potential [36] and related many-body potentials. The Stillinger-Weber potential includes the bond-bending three-body term needed to stabilize the relatively open diamond-cubic structure of the silicon crystal lattice:

$$
\begin{aligned}
U^{M D} & =\sum_{\mu>\nu} V_{2}\left(r_{\mu \nu}\right)+\sum_{\lambda>\mu>\nu} V_{3}\left(\mathbf{r}_{\lambda}, \mathbf{r}_{\mu}, \mathbf{r}_{\nu}\right) \\
V_{2}\left(r_{\mu \nu}\right) & =A \varepsilon\left(B \tilde{r}_{\mu \nu}^{-4}-1\right) \exp \left[1 /\left(\tilde{r}_{\mu \nu}-a\right)\right] \\
V_{3}\left(\mathbf{r}_{\lambda}, \mathbf{r}_{\mu}, \mathbf{r}_{\nu}\right) & =\lambda \varepsilon \exp \left[\gamma /\left(\tilde{r}_{\lambda \mu}-a\right)+\gamma /\left(\tilde{r}_{\mu \nu}-a\right)\right]\left(\cos \theta_{\lambda \mu \nu}+\frac{1}{3}\right)^{2}+\text { cyclic permutations }
\end{aligned}
$$

where the normalized interatomic distance is given by $\tilde{r}=r / \sigma$. Both terms go smoothly to zero at a distance $r=a \sigma$ and vanish for $r>a \sigma$. The values of the parameters are given in the original reference [36]; for our purposes we note that $a$ is chosen to give a cutoff between the first and second nearest neighbors in equilibrium at ambient conditions. 
The cosine of the bond angle is given by

$$
\cos \theta_{\lambda \mu \nu}=\frac{\mathbf{r}_{\mu \lambda} \cdot \mathbf{r}_{\mu \nu}}{r_{\mu \lambda} r_{\mu \nu}}
$$

where, for example, $\mathbf{r}_{\mu \nu}$ is the vector giving the relative position of atom $\mu$ with respect to atom $\nu$, and $r_{\mu \nu}$ is the corresponding distance. Note that $\cos \theta_{\lambda \mu \nu}=-\frac{1}{3}$ gives the minimum in energy and corresponds to the angle of the tetrahedral bonds in silicon. In BCC metals, we have used a different many-body potential due to Finnis and Sinclair. [34]

In $\mathrm{MD}$, once the interatomic potential is determined, the basic formulation immediately follows. Forces on each atom are determined from the potential, $\mathbf{F}_{\mu}=-\nabla_{\mathbf{r}_{\mu}} U$ where $\mathbf{F}_{\mu}$ is the force on the $\mu^{t h}$ atom, $\nabla_{\mathbf{r}_{\mu}}$ is the gradient with respect to the coordinate of the $\mu^{t h}$ atom and $U$ is the total potential energy; i.e. the sum of the many-body terms described above. Analytic derivatives are used for the potentials described here. The atomic motion due to these forces is described by Newton's third law

$$
m_{\mu} \ddot{\mathbf{r}}_{\mu}=-\nabla_{\mathbf{r}_{\mu}} U
$$

and in practice the motion is determined numerically using a time integrator such as the velocity Verlet algorithm. [25] Velocity Verlet integration is an example of a sympletic time integrator, and it allows longer time steps for a given level of error in the energy. Since one basic application of MD is to simulate motion in the microcanonical ensemble, energy conservation is important. In other cases, thermostats and barostats are added to simulate constant temperature and pressure.

Molecular dynamics has been used in some cases to simulate systems as large as $10^{9}$ atoms. [37] Since the interatomic potentials typically do not extend past the first few neighbor shells, the MD interactions are fairly local and MD is well suited to parallelization on supercomputers. The MD time step is tied to the characteristic frequencies of oscillation of the atoms in order to maintain accuracy with the direct time integration. For silicon, a time step of $0.5 \times 10^{-15} \mathrm{~s}$ is typical. The total time simulated is then limited to a few nanoseconds.

Finite Element Modeling. In the hybrid CLS model, MD is coupled to continuum solid mechanics implemented in linear FEM. Finite element modeling is one approach to discretization of partial differential equations to put them in a form suitable for solution on a computer. The discretization is done through the introduction of an irregular mesh. The fields of interest are defined by their values at the nodes (vertices) of the mesh, and shape functions are used to interpolate between the nodes. Consider Hooke's Law of continuum elasticity

$$
\sigma_{a b}=C_{a b c d} \varepsilon_{c d}
$$

which relates the stress $\sigma_{a b}$ to the strain $\varepsilon_{c d} \approx \frac{1}{2}\left(\partial_{c} u_{d}+\partial_{d} u_{c}\right)$ due to the displacement field, $\mathbf{u}(\mathbf{x})$. The elastic constants $C_{a b c d}$ relate the stress and strain. Note that here, and in the following, a sum is implied over repeated indices appearing on one side of an equation. The corresponding elastic potential energy is

$$
U^{\text {elastic }}=\int d^{3} x \frac{1}{2} C_{a b c d} \partial_{a} u_{b} \partial_{c} u_{d}
$$

with additional terms due to body forces, constrained boundaries and surface tractions. Let us introduce the shape function $N_{i}(\mathbf{x})$ associated with node $i$, so that the displacement field is given by

$$
\mathbf{u}(\mathbf{x})=\sum_{i} \mathbf{u}_{i} N_{i}(\mathbf{x})
$$

Typically, $N_{i}(\mathbf{x})$ is equal to unity at node $i$ and it decreases smoothly to zero at the neighboring nodes. In the work we describe below, $N_{i}(\mathbf{x})$ takes one of three forms. In three dimensions, we either use a mesh composed of tetrahedral elements with interpolation by linear shape functions or a mesh composed of hexahedral (distorted cubic) elements with interpolation by tri-linear shape functions (8-node bricks). We have used the tetrahedral elements for semiconductors for which it is difficult to tessellate the atomic lattice with hexahedra; we have used the hexahedral elements for metals. In some cases, we have also used a two dimensional triangular mesh with interpolation by linear shape functions [27] if the periphery can be assumed to be in plane stress. When Eq. (9) is substituted into the equation for the elastic potential energy (8), what results is the FEM expression for the potential energy:

$$
\begin{aligned}
U^{F E} & =\sum_{I} U_{I}^{F E} \\
U_{I}^{F E} & =\frac{1}{2} K_{i a j b ; I} u_{i a} u_{j b} \\
K_{i a j b ; I} & =C_{a c b d} \int_{\Omega_{I}} d^{3} x \partial_{c} N_{i}(\mathbf{x}) \partial_{d} N_{j}(\mathbf{x})
\end{aligned}
$$


where the integral in the definition of the stiffness matrix $K_{i a j b}$ is over the volume $\Omega_{I}$ of element $I$. This integral may be computed analytically in some cases, or through numerical quadrature more generally (we use multi-point Gaussian quadrature for the 8-node brick). The stiffness matrix is assembled element by element for efficiency, so that even though there is a global stiffness matrix given by $K_{i a j b}=\sum_{I} K_{i a j b ; I}$, in practice the energies and forces are computed element by element and this sum is never taken. We note that a more general approach to the derivation of FEM equations involves using the Galerkin approach on the weak form of the problem, but for linear elasticity the results are the same. [26] Substitution of the interpolated displacement field into the kinetic energy yields the FEM mass matrix

$$
\begin{aligned}
E_{k i n}^{F E} & =\frac{1}{2} M_{i j} \dot{\mathbf{u}}_{i} \cdot \dot{\mathbf{u}}_{j} \\
M_{i j} & =\int d^{3} x \rho(\mathbf{x}) N_{i}(\mathbf{x}) N_{j}(\mathbf{x})
\end{aligned}
$$

where $\rho$ is the mass density. This results in a set of equations of motion

$$
M_{i j} \ddot{u}_{j a}=-K_{i a k b} u_{k b}
$$

in the absence of external forces. In order to compute the evolution of the system, Eq. (15) must be solved for $\ddot{u}_{J i}$, which may then be used in a suitable time integration algorithm, such as the explicit velocity Verlet time integrator (although other time integrators are typically used in conventional FEM). It is convenient to replace the full distributed mass matrix (14) with a diagonal approximate mass matrix known as the lumped mass matrix

$$
\begin{aligned}
M_{i j}^{\text {lump }} & =M_{i}^{\text {lump }} \delta_{i j} \\
M_{i}^{\text {lump }} & =\rho \sum_{I \ni i} \frac{1}{\nu_{I}} V_{I}
\end{aligned}
$$

where $V_{I}$ is the volume of element $I$ with $\nu_{I}$ nodes, and the sum runs over elements with $i$ as a vertex. Then the equations of motion become

$$
M_{i}^{\text {lump }} \ddot{u}_{i a}=-K_{i a j b} u_{j b}
$$

and are of the same form as the MD equations of motion.

Once again, we emphasize that the MD and FEM formalisms are presented here to make the Article more selfcontained for readers who may not be familiar with both kinds of simulation. We have explained some of the choices we have made, but in large part the formulations we use are conventional. The interested reader is referred to the textbooks [25-27] and the references therein for more details.

Hybrid Coupling of Length Scales. We now turn to the question of how MD and FEM are combined in the hybrid CLS model. Since the equations of motion (6) and (18) are of the same form, they may be naturally combined in the same code. They both correspond to conserved energies, and since energy conservation is important to the statistical fidelity of MD, we have chosen to base the FEM-MD coupling on a unified hybrid Hamiltonian (energy) that is conserved. The Hamiltonian includes both the kinetic and potential energy. Consider first the kinetic energy, in which the unification of MD and FEM is easily accomplished. In both MD and lumped-mass FEM, the mass is associated directly with the atoms and nodes. The kinetic energy is just a sum of the kinetic energies of the atoms and the kinetic energies of the nodes. There is no need for an explicit coupling of the MD and FEM regions within the kinetic energy.

Now consider the potential energy, which does require an explicit coupling between MD and FEM. The coupling is implemented in the form of a handshaking Hamiltonian. [14, 17] Consider an imaginary fiducial surface running through the system defining the interface between the MD region on one side and the FEM region on the other. If we imagine extending the MD and FEM regions past this surface, there would be both MD interatomic bonds and FEM cells that cross the surface. The HS Hamiltonian is taken to be, in a precise sense defined below, the average of these MD and FEM potential energies at the interface. [14] The nodes of the FEM mesh are taken to be collocated with the equilibrium position of the MD atoms in the vicinity of the MD/FEM interface, and the crystal structure is forced to remain coherent throughout the simulation (i.e., no diffusion and no defects are allowed in the HS region). The value of the displacement at a node is identically equal to the displacement of the corresponding atom at all times. Thus, we may refer to the atoms and nodes in this HS region interchangeably. The HS Hamiltonian is taken to be the sum 
of all of these interactions, MD bond energies and FEM element energies, that pass through the fiducial surface at the FEM/MD interface, divided by two in order to average the MD and FEM contributions:

$$
U^{H S}=\frac{1}{2} \sum_{\mathbf{r}_{\mu \nu} \in H S} V_{2}\left(r_{\mu \nu}\right)+\frac{1}{2} \sum_{\mathbf{r}_{\lambda \mu}, \mathbf{r}_{\mu \nu} \in H S} V_{3}\left(\mathbf{r}_{\lambda}, \mathbf{r}_{\mu}, \mathbf{r}_{\nu}\right)+\frac{1}{2} \sum_{I \in H S} U_{I}^{F E}
$$

where the sums run over those elements and bonds that cross the fiducial surface. For the Stillinger-Weber potential this is all that is required. The potential, while complicated by its many-body nature, is relatively simple from the point of view of the range of its interactions. Only nearest neighbor atoms interact in the pair potential and in each leg of the 3-body interaction. The two- and three-body forces go to zero separately for the equilibrium diamond-cubic lattice at zero temperature. Thus, the HS region is relatively thin and there are no 'ghost' forces [38] that drive the MD/FEM interface to reconstruct, or exhibit other pathologies. The thinness of this interface is what we have referred to as minimal coupling. [14] Additional considerations are needed for interatomic potentials of longer range.

We have now completely defined the CLS Hamiltonian:

$$
H^{C L S}=\frac{1}{2} \sum_{\mu \in M D} m_{\mu}\left|\dot{\mathbf{r}}_{\mu}\right|^{2}+\frac{1}{2} \sum_{j \in F E} M_{j}^{\text {lump }}\left|\dot{\mathbf{u}}_{j}\right|^{2}+U^{M D}+U^{F E}+U^{H S}
$$

with $U^{M D}, U^{F E}$ and $U^{H S}$ defined by Eqs. (2), (10) and (19), respectively. The Euler-Lagrange equations for this Hamiltonian describe the motion of the atoms and nodes:

$$
\begin{aligned}
\ddot{\mathbf{r}}_{\mu} & =-\nabla_{\mathbf{r}_{\mu}}\left(U^{M D}+U^{H S}\right) / m_{\mu} \\
\ddot{\mathbf{u}}_{j} & =-\nabla_{\mathbf{u}_{j}}\left(U^{F E}+U^{H S}\right) / M_{j}^{\text {lump }}
\end{aligned}
$$

We integrate these equations using the velocity Verlet algorithm with the same time step for all degrees of freedom.

The NEMS resonators we have modeled contain up to several million atoms in the MD region, and the simulation must be run for several million time steps to obtain one period of oscillation of a silicon gigahertz resonator. The computational expense of these simulations is large, and so they have been run in parallel on many processors of a supercomputer. The NEMS resonator geometry is convenient from the point of view that a relatively simple onedimensional domain decomposition may be used, as shown in Fig. 3. The Message Passing Interface (MPI) is used for communications between processors on the distributed memory machine. The coordinates of the atoms within the range of the interatomic potential of neighboring domains are passed using MPI to that domain. Communications take place at each time step in order for the forces and energies to be computed. This parallel processing makes it possible to simulate the motion of multi-million atom systems for nanoseconds.

\section{CGMD: An atomistic/continuum effective model}

The hybrid approach embodied in the CLS methodology has the advantage that most of the simulation is run under one of two conventional models: molecular dynamics and finite element modeling. These techniques have been thoroughly studied and optimized. The only potential difficulty is in the FEM/MD interface, and to some extent in the region near the interface where the FEM mesh is refined nearly to the atomic level. In the CLS approach, as well as in other concurrent approaches such as the Quasicontinuum technique [21], it is acknowledged that the forces and/or energies are not quite right at the interface. The goal is to ensure that whatever errors are generated there do not affect the calculation of the properties and system behavior of interest. For example, in the case of NEMS resonators, the errors are tolerable as long as they do not cause an appreciable shift in the resonator frequency or a measurable change in its quality factor. The magnitude of these errors can often be estimated, for example by testing for sensitivity to the location of the FEM/MD interface (the results should not change as the interface is moved farther into the periphery). However, it may be necessary, and in any case it is desirable, to have a technique in which the fidelity of the interface may be made as high as possible.

The use of effective concurrent multiscale modeling, such as embodied in CGMD, is one way to ensure the fidelity of the interface and to control any errors that arise there. In CGMD, conventional finite element modeling is not used. Instead, a systematic coarse-graining procedure based on statistical mechanics is used to develop an effective solid mechanics model on the irregular mesh. Once again, we focus our attention on the Hamiltonian, the conserved energy from which we are able to derive the equations of motion. In FEM, the Hamiltonian was derived from a discrete representation of the continuum elastic fields. CGMD does not make use of continuum elasticity. One of the 


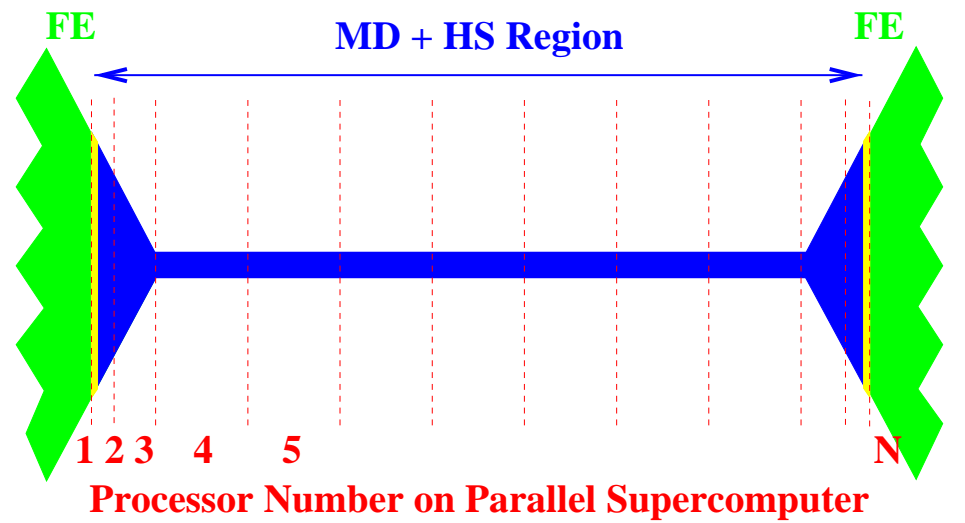

FIG. 3: A schematic representation of the domain decomposition of the system across processors of a parallel supercomputer, showing which atoms are computed on which processors. The MD calculations are substantially more expensive computationally, so most of the processors are used for MD calculations (and hence this diagram is focussed on the MD region). At each time step the coordinates of those atoms outside a given domain, but outside by less than the range of the interatomic potential, are communicated to that domain for the purposes of the force and energy calculations. The parallel processing gives a substantial reduction in the wall-clock time needed for the simulation of million atom systems. [15]

problems with FEM for concurrent multiscale modeling is that an atomistic model does not occur naturally as the mesh is refined to the atomic level. [39] Continuum elasticity does not know about the discrete atoms; in fact the basic premise of FEM, that the kinetic and elastic potential energies are smoothly interpolated within each element of the mesh, is violated at the atomic scale. The kinetic energy is localized in the atomic nuclei and the potential energy is localized in the covalent bonds. Mathematically, continuum elasticity results from taking the large-N or thermodynamic limit of a system containing $\mathrm{N}$ atoms. Corrections to the large- $\mathrm{N}$ limit form an asymptotic series in $1 / \mathrm{N}$ [40] and recovering a unique finite $\mathrm{N}$ theory from the asymptotic limit is almost always impossible. The statistical coarse-graining procedure of CGMD avoids this problem by deriving the discrete mesh-based model directly from the discrete atomistic model.

In CGMD, an interpolated displacement field is introduced, $\mathbf{u}(\mathbf{x})=\sum_{i} \mathbf{u}_{i} N_{i}(\mathbf{x})$, but it is not used to calculate the continuum elastic energy. Instead, it is used to constrain the motion of the atoms governed by the MD energy. A constraint is imposed that interpolated displacement field should be a best fit to the atomic displacements from their equilibrium lattice sites. In particular, the chi-squared error should be minimized with respect to changes in $\mathbf{u}_{i}$ :

$$
\begin{aligned}
\chi^{2} & =\sum_{\mu}\left|\mathbf{u}_{\mu}-\mathbf{u}\left(\mathbf{r}_{\mu 0}\right)\right|^{2} \\
& =\sum_{\mu}\left|\mathbf{u}_{\mu}-\sum_{i} \mathbf{u}_{i} N_{i \mu}\right|^{2}
\end{aligned}
$$

where $\mathbf{r}_{\mu 0}$ is the equilibrium lattice site of atom $\mu$ and we have introduced the atomic displacement $\mathbf{u}_{\mu} \equiv \mathbf{r}_{\mu}-\mathbf{r}_{\mu 0}$. We have also introduced notation for the discretized interpolation function:

$$
N_{i \mu}=N_{i}\left(\mathbf{r}_{\mu 0}\right)
$$

The minimum of chi-squared is found by setting the derivative $\partial_{\mathbf{u}_{i}} \chi^{2}$ equal to zero and solving for $\mathbf{u}_{i}$ :

$$
\mathbf{u}_{i}=\left(N_{i \mu} N_{j \mu}\right)^{-1} N_{j \nu} \mathbf{u}_{\nu} \equiv f_{i \lambda} \mathbf{u}_{\lambda}
$$

where the inverse is a matrix inverse. This gives a total of $3 N_{\text {node }}$ constraints on the positions of the $3 N_{a t o m}$ atoms; there are another $3 N_{\text {node }}$ constraints on the velocities of the atoms as well. So of the total $6 N_{\text {atom }} \mathrm{MD}$ degrees of freedom, only $6\left(N_{\text {atom }}-N_{\text {node }}\right)$ degrees of freedom are unconstrained and free to fluctuate. [41] Of course, the mesh may be much larger than the atomic scale at the periphery, so $N_{\text {atom }} \gg N_{\text {node }}$, and these fluctuating degrees of freedom may be treated with statistical mechanics. 
We define

$$
H^{\mathrm{CGMD}}\left(\mathbf{u}_{i}, \dot{\mathbf{u}}_{j}\right)=\int \prod_{\mu}\left(d^{3} r_{\mu} d^{3} p_{\mu}\right) H^{\mathrm{MD}} e^{-H^{\mathrm{MD}} / k T} \Delta / Z
$$

where $\Delta$ is a product of delta functions to enforce the constraint (26) and the corresponding constraint relating the atomic momenta to the nodal velocities. The integral is divided by the partition function, $Z$, which is given by the same integral without the pre-exponential factor of $H^{\mathrm{MD}}$. The calculation of this integral over a $6 N_{\text {atom }}$ dimensional space has not been possible in general. The space is too large for numerical techniques, and the non-linear MD potential prohibits a general analytic solution; however, an analytic calculation is possible if the MD potential energy is harmonic. For solids well below their melting temperature, quasi-harmonic lattice dynamics provides a good description. [42, 43] This suggests that the CGMD energy (27) may be calculated approximating the solid as a harmonic lattice, and then calculating corrections in perturbation theory. Both the harmonic CGMD energy and the perturbative corrections have been calculated exactly with analytic techniques. [18, 20]

The result is an expression for the CGMD Hamiltonian of the following form [18]:

$$
H^{\mathrm{CGMD}}\left(\mathbf{u}_{i}, \dot{\mathbf{u}}_{j}\right)=3\left(N_{a t o m}-N_{n o d e}\right) k T+U_{0}+\frac{1}{2} M_{i j}^{\mathrm{CGMD}} \dot{\mathbf{u}}_{i} \cdot \dot{\mathbf{u}}_{j}+\frac{1}{2} K_{i a j b}^{\mathrm{CGMD}} u_{i a} u_{j b}+\cdots
$$

assuming no external body forces, tractions, etc. The first two terms represent internal energy contributions coming from the $6\left(N_{\text {atom }}-N_{\text {node }}\right)$ modes that have been integrated out, the cohesive energy, and so on. The next two terms are the kinetic and elastic potential energies of the modes supported by the mesh. Note that they are of the same form as the FEM energies (11) and (13). All of these terms come from the harmonic lattice. The ellipses denote anharmonic contributions calculated in perturbation theory. [20]

While Eq. (28) shares the same form as the FEM energy, there are important differences in expressions for the mass and stiffness matrices. [18] The CGMD mass and stiffness matrices for a monatomic lattice are given by

$$
\begin{aligned}
M_{i j}^{\mathrm{CGMD}} & =m N_{i \mu} N_{j \mu} \\
\mathbf{K}_{i j}^{\mathrm{CGMD}} & =\left(f_{i \mu} \mathbf{D}_{\mu \nu}^{-1} f_{j \nu}\right)^{-1}
\end{aligned}
$$

where $m$ is the atomic mass and the inverses are matrix inverses. We have suppressed the spatial indices in the stiffness formula. The mass matrix is an average of the atomic masses, and the stiffness matrix is an average of the atomic dynamical matrix; i.e., the second order (harmonic) term in the expansion of interatomic potential energy. The inverse of the dynamical matrix, $D_{\mu a \nu b}$, is calculated in reciprocal space; otherwise, the matrix inverse would be prohibitive. Recent advances in the understanding of the stiffness matrix have further facilitated its calculation. [20]

It has been shown that the CGMD equations of motion in regions of the mesh refined to the atomic scale are just the usual MD equations of motion (6). [18] Thus, in CGMD it is still convenient to distinguish between two regions of the simulation: the $\mathrm{MD}$ region, where the nodes and atoms are in one-to-one correspondence, and the coarse-grained (CG) region. The equivalence of fine-grained CGMD and MD follows from the fact that in the MD region $f_{i \lambda}=\delta_{i \lambda}$, so the constraint (26) is simply that $\mathbf{u}_{i}=\mathbf{u}_{\lambda}$. The delta function constraint in the expression for the CGMD Hamiltonian then just returns $H^{\mathrm{MD}}$ for those atoms that are well inside the MD region (by more than the range of the MD potential). The equation of motion for the atoms in the MD region is just the usual MD equation of motion based the usual empirical many-body potential. The perturbation expansion is not used there. In the CG region the equation of motion derived from the CGMD Hamiltonian (27) is of the finite element form:

$$
M_{i j} \ddot{u}_{j a}=-K_{i a k b} u_{k b}+\ldots
$$

Again, the ellipses indicate additional non-linear terms arising from anharmonic forces. [14, 19] This set of equations is solved for $\ddot{\mathbf{u}}_{j}$, which is then used in the velocity Verlet algorithm to evolve the system.

Coarse-grained molecular dynamics has proven very effective in permitting large volumes of the system to be simulated with a reduced number of degrees of freedom. This allows the modeling of larger systems sizes than would otherwise be possible with the given computer resources. Also, CGMD has certain advantages over a straight finite element/molecular dynamics hybrid approach. Conventional finite element models do not automatically give the MD equation of motion as the cell size is reduced to the atomic scale; CGMD does. The result is a higher quality interface between the MD and CG regions. Unwanted elastic wave scatter is reduced, although not completely eliminated (see the discussion below). [14] The elastic wave spectrum is better represented in the CG region. Effects such as thermal expansion are treated properly. [20] CGMD is more expensive computationally than conventional finite element modeling, but in some instances this additional cost is warranted in order to achieve a higher quality simulation. 
The original formulation of CGMD is a Hamiltonian system. Thermal effects are present, but not the random, dissipative forces due to the heat bath of modes that have been integrated out. Energy is conserved. Recently, a non-conservative version of CGMD has been developed [19] in order to remove unphysical elastic wave reflection. We note that this problem has been addressed with other techniques as well. [44, 45] In a conservative framework, elastic waves are reflected from regions of the mesh where the mesh spacing exceeds the wave length. [18] Basically, energy is conserved, so the energy in the wave must go somewhere. If the mesh spacing is too large, the wave cannot be supported on the mesh: it is reflected. Projection operator techniques have been used to derive a generalized Langevin equation that solves this problem. [19]

To date, CGMD has primary been used as a standard to judge the efficacy of other concurrent multiscale methodologies, and to guide in their development. CGMD is more expensive computationally than the hybrid CLS technique, and it has not been possible to use it in large simulations (for example, we have used CLS in simulations with over 100,000 nodes in the FEM region). Some progress on the efficiency of CGMD has been made recently [20], and there is additional margin for improvement. Without the possibility of large-scale simulations, CGMD has been used in some interesting benchmarks for multiscale simulation. Phonon spectra have been calculated in CGMD and compared with the spectra of the underlying MD model and those of several formulations of FEM, initially for a one-dimensional chain of atoms [18], and then for a three-dimensional solid argon crystal. [14, 20] The result was that CGMD agreed with the MD spectrum in the limit of an atomic-size mesh, whereas there were significant inaccuracies in the FEM calculations. In the limit of large mesh sizes, CGMD once again out-performed the FEM, but of course none was exact in this limit.

\section{ATOMISTIC EFFECTS IN NEMS}

The conventional description of the mechanical components of MEMS is based on continuum mechanics implemented in a finite element model. [26] The oscillations of a resonator, for example, are dictated by the geometry of the system, the properties of the materials and the applied forces. In the linear elastic theory of macroscopic beams [46] such as flexural resonators, the material properties are just the elastic constants and the mass density of the material. These properties are taken to be independent of size, an assumption that is valid as long as the variations of the elastic fields occur over lengths that are large compared to the characteristic lengths of any microstructure. For example, the material properties would differ for a resonator a millimeter thick and one a micron thick, if the material has a 100 nm oxide layer. Indeed, the material could exhibit fatigue if the oxide layer changes in time due to stress or cycling. $[47]$

We are primarily interested in NEMS bulk micromachined from a single crystal semiconductor (silicon). At first glance, this appears to be a homogeneous system, and the material properties should be scale independent. This is not entirely the case, however, because the lattice constant (the interatomic spacing) sets a scale. The width of the smallest NEMS resonators is on the order of 100 atoms wide, so the properties of the resonator can be affected by the discrete, atomic lattice.

Another issue is the fact that the surface area-to-volume ratio increases as systems are miniaturized, and surface effects become more important. This fact has long been appreciated in the MEMS community. [48] It takes on new significance for NEMS. Surface effects can take many forms. The first example we consider is the effect of surfaces on NEMS elastic properties. One of the results from our work and that of our collaborators is that the Young's modulus, the elastic constant that enters the resonant frequency of a flexural resonator, becomes scale dependent due to the contribution of surface stress in addition to the bulk elastic constants (cf. Fig. 4). Such a result is expected if the surface is heavily contaminated or chemically different that the bulk (e.g. due to oxidation), but we find the surface contribution even in simulations of perfectly clean, pure and defect-free resonators. The surface effect is very general, applying to every material we have tried so far: $\alpha$-quartz [15, 49], silicon [50] and tantalum [50] (see also [51]). At the level of atomistic physics, the renormalization of the modulus occurs because the surface atoms are bonded differently than the bulk atoms. In some cases the lattice structure may reconstruct at the surface. Silicon is an excellent example, where the diamond cubic lattice changes to a $2 \times 1$ dimer-row reconstruction on the (100) surface. The reconstruction changes the configuration of the bonds, so the stiffness of the surface atoms is different than that of the bulk. Even without the reconstruction, the surface stiffness may differ. Again a good example is silicon, where the unreconstructed surface is metastable at absolute zero temperature. The Young's modulus in the metastable unreconstructed state still exhibits a size effect. [50] In the quartz simulations, the surfaces were oxygen terminated, and again they exhibited a different stiffness than the bulk. [15, 49] In the metallic NEMS bar made of tantalum, the calculated Young's modulus again showed a surface effect. In this case, the lower coordination of the surface atoms led to stronger bonds and hence an increase in the stiffness. [50] 


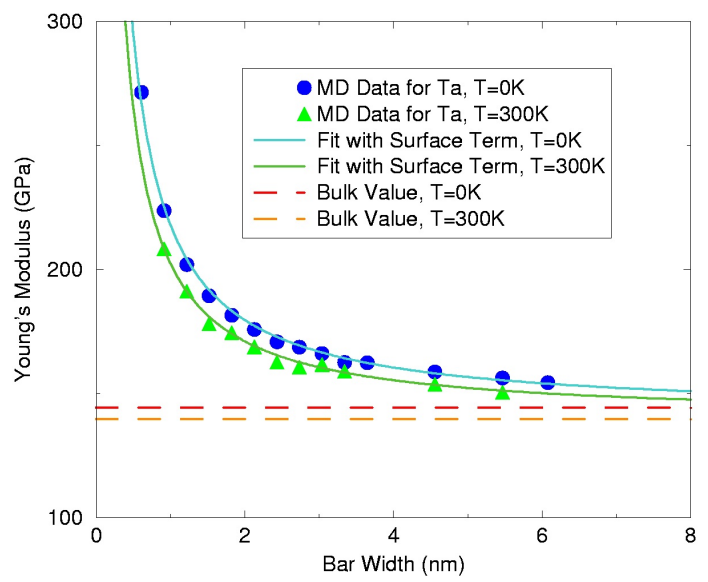

FIG. 4: Young's modulus vs. thickness for a tantalum nanobeam calculated with MD at zero temperature and room temperature, as indicated. The solid curves are the chi-squared fit to a simple model including surface stress. The dashed curves indicate the value of the Young's modulus for a bulk system, also calculated in MD at the indicated temperatures. This size effect is an example of the renormalization of mechanical properties at the nanoscale due to surface effects. [50]

The basic result is that for a resonator that is 100 atoms wide, the computed deviation from the continuum Young's modulus is about $1 \%$. It increases further in our calculations as the system size is decreased, but this $(\sim 50 \mathrm{~nm})$ is the limit of the current e-beam lithography fabrication techniques. A direct verification of this $1 \%$ change is not possible due to other uncertainties in the fabrication process. It is possible to measure shifts in the resonant frequency of a single device that are much less than $1 \%$, so an indirect measurement may be possible. Recently, a direct mechanical measurement of the modulus of nanowires has been made in which size effects were reported. [52] The length scale of these effects was larger than what we have found in MD simulations of NEMS resonators, and the authors attribute the measurement to another kind of surface effect. Their results are not yet a confirmation of the MD predictions, but their measurement indicates that direct mechanical measurements of the native surface contribution to the elastic moduli may soon be possible.

The second aspect of NEMS behavior that we have investigated is dissipation. Dissipation leads to a degradation of the quality factor of the resonator, $Q$, that determines the width of the frequency response peak at resonance. A high $Q$ corresponds to a narrow peak. High $Q$ is desirable for applications like frequency standards and filters. The uncertainty in the frequency $\omega$ is $\delta \omega=\omega / Q$, so high $Q$ gives more precise control of the frequency. [6] Scaling arguments predict that sub-micron bridge-type oscillators could be made to resonate in the $\mathrm{GHz}$ regime, something that would be very useful for communications applications. [1, 6] Most ultra-high frequency NEMS resonators, and indeed other types of gigahertz resonators, typically have a very low $Q$, but the use of single crystal NEMS resonators would eliminate many dissipative processes [53] and hold the hope of relatively high $Q$ values. In fact, our simulations $[14,15]$ have found that $Q$ decreases as devices are miniaturized even for perfect single crystal devices: the dissipation, $1 / Q$, scales roughly like the surface area-to-volume ratio. This effect has been observed in many experiments as well. [7, 54-72] Recently it was reported that 1 GHz NEMS flexural mode resonators have been successfully fabricated. [70] The $1 \mathrm{GHz}$ resonators have a low quality factor, $Q \sim 500$, although the cause for this has not yet been explained.

We have investigated dissipation using atomistic and multiscale simulations. The results from two such simulations are shown in Fig. 5. [15] The curves represent the oscillations of systems of two different sizes with the same aspect ratio. The two systems were 0.02 microns and 0.14 microns long and contained 20 thousand and 2 million atoms in the MD region, respectively. The larger system exhibits longer period oscillations, corresponding to a frequency of about $2 \mathrm{GHz}$. The smaller system has a resonant frequency of about $24 \mathrm{GHz}$. The oscillations of the larger system are sinusoidal throughout the 1 ns simulation time, whereas the oscillations of the smaller system begin sinusoidally, but rapidly degenerate into a noisy motion. This degeneration is the result of dissipation. Energy is transferred from the fundamental mode into the other normal modes of the oscillator, as evident in the Fourier transform of the oscillations. [15] 


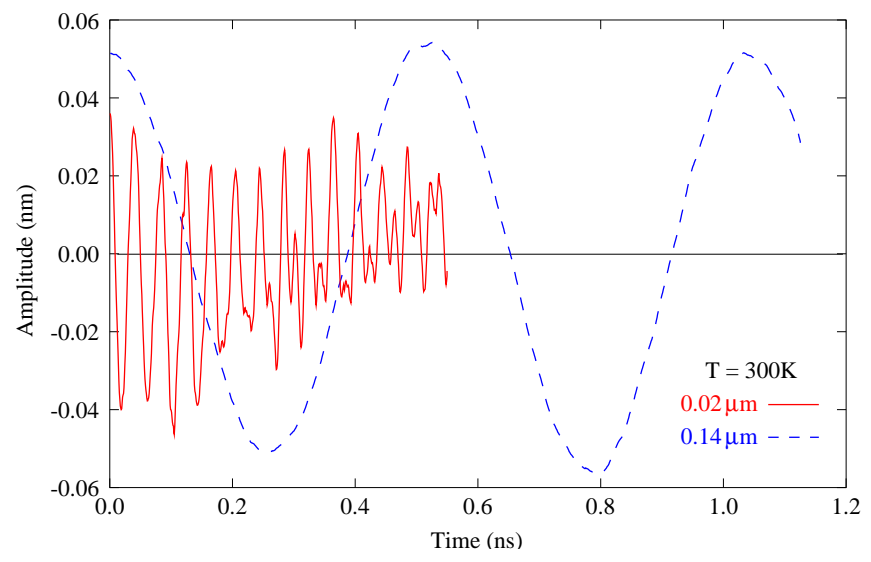

FIG. 5: Comparison of the oscillations of resonators of two different sizes, 0.02 microns and 0.14 microns long, but with the same aspect ratio. Both are at room temperature. Note the increased dissipation in the smaller resonator. This is due to a mode-mixing effect coming from lattice anharmonicity. [14, 15]

\section{CONCLUSIONS AND OUTLOOK}

There are of course many other issues that remain to be explored in the mechanics and dynamics of NEMS and many additional applications for concurrent multiscale modeling. With regard to NEMS resonators, it is still an open question what the dominant source of dissipation is in the current experiments and what the ultimate source of dissipation will be. The systems we have simulated are ideal in many ways. They are monatomic or diatomic (quartz), without any impurities or defects. It has been hypothesized in the literature [66, 73-75] that two-level systems of the sort found in glassy materials are responsible for most of the dissipation, but the nature of the two-level systems has not been determined. If it is a disordered layer of adsorbates on the surface of the device, it would be difficult to obtain enough structural information to conduct a realistic simulation of the system at the atomistic level. Nevertheless, it may be possible to use a simplified model of the layer within a concurrent multiscale simulation to gain some insight into candidate mechanisms. One group has reported that the dissipation shows a strong dependence on magnetic field. [66] This suggests that electronic processes may be dominant, which unfortunately are beyond the scope of the techniques we have presented.

Concurrent multiscale techniques have been used for a variety of other applications, including plasticity [21, 22, 76], fracture $[17,23]$, and nanoindentation $[77,78]$. This list is certainly not comprehensive, and the number of applications treated with concurrent multiscale modeling is growing steadily. We are just at the start of what promises to be a very interesting development.

\section{Acknowledgments:}

We gratefully acknowledge the important contributions of Jeremy Broughton, with whom this work was begun. We thank DARPA for initial funding of the MEMS research, and we thank LLNL for funding of the nanomechanics work under the Laboratory Directed Research and Development program. This work was performed in part under the auspices of the U.S. Department of Energy by the University of California, Lawrence Livermore National Laboratory, under Contract No. W-7405Eng-48.

* E-mail: robert.rudd@llnl.gov

[1] A. N. Cleland and M. L. Roukes, Appl. Phys. Lett. 69, 2653 (1996); See also http://www.cmp.caltech.edu/ roukes/images/mrfm.jpg.

[2] M. Madou, Fundamentals of Microfabrication: The Science of Miniaturization, (CRC Press, Boca Raton, 2002).

[3] G. T. A. Kovacs, Micromachined Transducers Sourcebook, (McGraw-Hill, Boston, 1998).

[4] G. Wu, R. H. Datar, K. M. Hansen, T. Thundat, R. J. Cote, and A. Majumdar, "Bioassay of prostrate-specific antigen (PSA) using microcantilevers," NatureBiotech. 19, 856 (2001).

[5] See for example Y. Yang, H. F. Ji and T. Thundat, "Nerve agents detection using a Cu2+/L-cysteine bilayercoated microcantilever," J. Amer. Chem. Soc. 125, 1124 (2003).

[6] C. T.-C. Nguyen, et al, Proc. IEEE 86, 1756 (1998). 
[7] D. W. Carr, S. Evoy, L. Sekaric, H. G. Craighead, and J. M. Parpia, "Measurement of mechanical resonance and losses in nanometer scale silicon wires," Appl. Phys. Lett. 75, 920 (1999).

[8] R. M. D. Stevens, et al., "Carbon Nanotubes as Probes for Atomic Force Microscopy," Nanotechnology 11, 1 (2000).

[9] M. B. Viani, et al., "Probing Protein-Protein Interactions in Real Time," Nature 7, 644 (2000).

[10] K. Schwab, E. A. Henriksen, J. M. Worlock and M. L. Roukes, "Measurement of the quantum of thermal conductance," Nature 404, 974 (2000).

[11] A. D. Armour, M. P. Blencowe and K. C. Schwab, "Entanglement and Decoherence of a Micromechanical Resonator Via Coupling to a Cooper-Pair Box," Phys. Rev. Lett. 88, 148301 (2002).

[12] A. Cho, "Researchers race to put the quantum into mechanics," Science 299, 36 (2003).

[13] See for example, Technical Proceedings of the 1998 International Conference on Modeling and Simulation of Microsystems, Semiconductors, Sensors and Actuators, April 6-8, 1998, Santa Clara, CA, M. Laudon and B. Romanowicz, eds., (Computational Publications, Boston, 1998).

[14] R. E. Rudd and J. Q. Broughton, "Concurrent Coupling of Length Scales in Solid State Systems," Phys. Stat. Sol. (b) 217, 251 (2000).

[15] R. E. Rudd and J. Q. Broughton, "Atomistic Simulation of MEMS Resonators through the Coupling of Length Scales," J. Model. and Sim. of Microsys. 1, 26 (1999).

[16] R. E. Rudd, "Concurrent Multiscale Modeling of Embedded Nanomechanics," Mater. Res. Soc. Symp. Proc. 677, AA1.6 (2001).

[17] J. Q. Broughton, N. Bernstein, E. Kaxiras, and F. F. Abraham, "Concurrent coupling of length scales: Methodology and application," Phys. Rev. B 60, 2391 (1999).

[18] R. E. Rudd and J. Q. Broughton, "Coarse-grained molecular dynamics and the atomic limit of finite elements," Phys. Rev. B 58, R5893 (1998).

[19] R. E. Rudd, "Coarse-grained molecular dynamics: Dissipation due to internal modes," Mat. Res. Soc. Symp. Proc. 695, T10.2.1-6 (2002), pp. 499-504.

[20] R. E. Rudd and J. Q. Broughton, "Coarse-grained molecular dynamics: nonlinear finite elements and finite temperature," in preparation for Phys. Rev. B (2003).

[21] E. B. Tadmor, M. Ortiz and R. Phillips, "Quasicontinuum Analysis of Defects in Solids," Phil. Mag. A73, 1529 (1996).

[22] L. E. Shilkrot, R. E. Miller, and W. A. Curtin, "Coupled Atomistic and Discrete Dislocation Plasticity," Phys. Rev. Lett, 89, 025501 (2002).

[23] S. Kohlhoff, P. Gumbsch and H. F. Fischmeister, "Crack propagation in bcc crystals studied with a combined finite-element and atomistic model," Phil. Mag. A 64, 851 (1991).

[24] S. Weinberg, Quantum Theory of Fields, (Cambridge University Press, Cambridge, 1995).

[25] M. P. Allen and D. J. Tildesley, Computer Simulation of Liquids, (Clarendon Press, Oxford, 1987).

[26] O. C. Zienkiewicz and R. L. Taylor, The Finite Element Method, 4th ed., (McGraw-Hill, London, 1991).

[27] T. J. R. Hughes, The Finite Element Method: Linear Static and Dynamic Finite Element Analysis, (Dover, Mineola, 2000).

[28] M. Finnis, Interatomic Forces in Condensed Matter (Oxford University Press, Oxford, 2003).

[29] We employ the following convention for indices. Atoms are indexed by Greek letters, $\mu, \nu, \ldots$. Nodes of the FEM mesh are indexed by lowercase Latin letters in the middle of the alphabet, $i, j, \ldots$ and elements (cells) of the mesh are indexed by uppercase Latin letters in the middle of the alphabet, $I, J, \ldots$ Spatial dimensions are indexed by Latin letters at the start of the alphabet, $a, b, \ldots$, but these indices will be suppressed whenever possible through the use of bold font to denote vectors.

[30] J. Grindlay and R. Howard, "On the Lattice Dynamics and Specific Heat of the Rare-Gas Solids," in Lattice Dynamics, Proc. Int. Conf. Copenhagen Denmark, R. F. Wallis, ed (Pergamon, Oxford, 1965), p. 129.

[31] H. Balamane, T. Halicioglu and W. A. Tiller, "Comparitive study of silicon empirical interatomic potentials," Phys. Rev. B 46, 2250 (1992).

[32] D. G. Pettifor and I. I. Oleinik, "Analytic bond-order potentials beyond Tersoff-Brenner," Phys. Rev. B 59, 8487 (1999).

[33] J. A. Moriarty, "Analytic representation of multi-ion interatomic potentials in transition metals," Phys. Rev. B 42, 1609 (1990); "Angular forces and melting-in BCC transition metals: a case study of molybdenum," Phys. Rev. B 49, 12431 (1994).

[34] M. Finnis and J. Sinclair, "A simple empirical N-body potential for transition metals," Philos. Mag. A 50, 45 (1984); G. J. Ackland and R. Thetford, "An improved N-body semi-empirical model for bcc transition metals," Phil. Mag. A, 56, 15 (1987). 
[35] M. Daw and M. Baskes, "Semiempirical, Quantum Mechanical Calculation of Hydrogen Embrittlement in Metals," Phys. Rev. Lett. 50, 1285 (1983).

[36] F. H. Stillinger and T. A. Weber, "Computer simulation of local order in condensed phases of silicon," Phys. Rev. B 31, 5262, (1985).

[37] F. F. Abraham, R. Walkup, H. Gao, M. Duchaineau, T. Diaz de la Rubia, M. Seager, "Simulating materials failure by using up to one billion atoms and the world's fastest computer: Work-hardening," Proc. Natl. Acad. Sci. USA, 99, 5783 (2002).

[38] W. A. Curtin and R. E. Miller, "Atomistic/Continuum Coupling in Computational Materials Science," Modelling Simul. Mater. Sci. Eng. 11, R33 (2003).

[39] R.E. Rudd, "The Atomic Limit of Finite Element Modeling in MEMS: Coupling of Length Scales," J. on Analog Integ. Circuits and Signal Proc. 29, 17 (2001).

[40] C. M. Bender and S. A. Orszag, Advanced Mathematical Methods for Scientists and Engineers, (McGraw-Hill, New York, 1978).

[41] Note that $N_{\text {atom }}$ is the number of atoms in the whole system, including those in the CG region. This is not to be confused with the number of atoms in the MD region.

[42] M. Born and K. Huang, Dynamical Theory of Crystal Lattices, (Clarendon Press, Oxford, 1954).

[43] D. C. Wallace, Thermodynamics of Crystals, (Dover, Mineola, 1972).

[44] B. L. Holian and R. Ravelo, Phys. Rev. B 51, 11275 (1995).

[45] W. Cai, M. de Koning, V. V. Bulatov and S. Yip, "Minimizing Boundary Reflections in Coupled-Domain Simulations," Phys. Rev. Lett. 85, 3213 (2000).

[46] S. P. Timoshenko, S. Woinowsky-Krieger, Theory of Plates and Shells, (McGraw-Hill, New York, 1959).

[47] C. L. Muhlstein, E. A. Stach and R. O. Ritchie, "A reaction-layer mechanism for the delayed failure of micron-scale polycrystalline silicon structural films subjected to high-cycle fatigue loading," Acta Mater. 50, 3579 (2002).

[48] See for example K. E. Drexler, Nanosystems: molecular machinery, manufacturing and computation, (Wiley, New York, 1992).

[49] J. Q. Broughton, C. A. Meli, P. Vashishta, and R. K. Kalia, "Direct atomistic simulation of quartz crystal oscillators: Bulk properties and nanoscale devices," Phys. Rev. B 56, 611 (1997).

[50] R. E. Rudd, in preparation 2003.

[51] R. E. Miller and V. B. Shenoy, "Size-dependent Elastic Properties of Nanosized Structural Elements," Nanotechnology 11, 139 (2000).

[52] S. Cuenot, S. Demoustier-Champagne, C. Frétigny, and B. Nysten, "Size effect on the elastic modulus of nanomaterials as measured by resonant contact atomic force microscopy," in Proc. Nanotech 2003, San Francisco, CA, Feb. 23-27, 2003, M. Laudon and B. Romanowicz, eds. (Computational Publications, Boston, 2003), Vol. 3, pp. $549-52$.

[53] V. B. Braginsky V. P. Mitrofanov and V. I. Panov, Systems With Small Dissipation, (Univ. Chicago Press, Chicago, 1985).

[54] D. S. Greywall, B. Yurke, P. A. Busch and S. C. Arney, "Low-temperature anomalies in the dissipation of small mechanical resonators," Europhys. Lett. 34, 37 (1996).

[55] R. D. Biggar and J. M. Parpia, "Magnetic field effects on boron-doped Si oscillators," Phys. Rev. B 56, 13638 (1997).

[56] S. Evoy, D. W. Carr, L. Sekaric, A. Olkhovets, J. M. Parpia, and H. G. Craighead, "Nanofabrication and electrostatic operation of single-crystal silicon paddle oscillators," J. Appl. Phys. 86, 6072 (1999).

[57] S. Evoy, A. Olkhovets, L. Sekaric, J. M. Parpia, H. G. Craighead, and D. W. Carr, "Temperature-dependent internal friction in silicon nanoelectromechanical systems," Appl. Phys. Lett. 77, 2397 (2000).

[58] J. Yang, T. Ono, and M. Esashi, "Surface effects and high quality factors in ultrathin single-crystal silicon cantilevers," Appl. Phys. Lett. 77, 3860 (2000).

[59] K. Y. Yasumura, T. D. Stowe, E. M. Chow, T. Pfafman, T. W. Kenny, B. C. Stipe, and D. Rugar, "Quality factors in micron- and submicron-thick cantilevers," J. Microelectromech. Syst. 9, 117 (2000).

[60] C. L. Spiel, R. O. Pohl, and A. T. Zehnder, "Normal mode of a Si(100)double-paddle oscillator," Rev. Sci. Inst. 72, 1482 (2001).

[61] J. Yang, T. Ono, and M. Esashi, "Investigating surface stress: Surface loss in ultrathin single-crystal silicon cantilevers," J. Vac. Sci. Technol. B 19, 551 (2001).

[62] Y. T. Yang, K. L. Ekinci, X. M. H. Huang, L. M. Schaivone, M. L. Roukes, C. A. Zorman, and M. Mehregany, "Monocrystalline silicon carbide nanoelectromechanical systems," Appl. Phys. Lett. 78, 162 (2001).

[63] X. Liu, S. F. Morse, J. F. Vignola, D. M. Photiadis, A. Sarkissian, H. M. Marcus, and B. H. Houston, "On the mode and loss mechanisms of a high Q mechanical oscillator," Appl. Phys. Lett. 78, 1346 (2001). 
[64] A. N. Cleland, M. Pophristic, and I. Ferguson, "Single-crystal aluminum nitride nanomechanical resonators," Appl. Phys. Lett. 79, 2070 (2001).

[65] B. H. Houston, D. M. Photiadis, H. M. Marcus, J. A. Bucaro, X. Liu, and J. F. Vignola, "Thermoelastic loss in microscale oscillators," Appl. Phys. Lett. 80, 1300 (2002).

[66] P. Mohanty, D. A. Harrington, K. L. Ekinci, Y. T. Yang, M. J. Murphy and M. L. Roukes, "Intrinsic dissipation in high-frequency micromechanical resonators," Phys. Rev. B 66, 085416 (2000).

[67] J. Yang, T. Ono, and M. Esashi, "Energy dissipation in submicrometer thick single-crystal silicon cantilevers," J. Microelectromech. Syst. 11, 775 (2002).

[68] X. Liu, J. F. Vignola, D. M. Photiadis, A. Sarkissian, B. H. Houston, R. D. Merithew, and R. O. Pohl, "Low temperature study of loss mechanisms of mechanical oscillators," Physica B 316, 393 (2002).

[69] D. M. Photiadis, B. H. Houston, X. Liu, J. A. Bucaro, and M. H. Marcus, "Thermoelectric loss observed in a high Q mechanical oscillator," Physica B 316, 408 (2002).

[70] X. M. H. Huang, C. A. Zorman, M. Mehregany, and M. L. Roukes, "Nanodevice motion at microwave frequencies," Nature 421, 496 (2003).

[71] T. Ono, D. F. Wang, and M. Esashi, "Time dependence of energy dissipation in resonating silicon cantilevers in ultrahigh vacuum," Appl. Phys. Lett. 83, 1950 (2003).

[72] K.-H. Ahn and P. Mohanty, "Quantum friction of micromechanical resonators at low temperatures," Phys. Rev. Lett. 90, 85504 (2003).

[73] R. N. Kleiman, G. Agnolet and D. J. Bishop, "Two-level systems observed in the mechanical properties of single-crystal silicon at low temperatures," Phys. Rev. Lett. 59, 2079 (1987).

[74] W. A. Phillips, "Comment on "Two-level systems observed in the mechanical properties of single-crystal silicon at low temperatures'," Phys. Rev. Lett. 61, 2632 (1988).

[75] R. W. Keyes, "Two-level systems in the mechanical properties of silicon at low temperatures," Phys. Rev. Lett. 62, 1324 (1989).

[76] V. B. Shenoy, R. V. Kukta and R. Phillips, "Mesoscopic Analysis of Structure and Strength of Dislocation Junctions in fcc Metals," Phys. Rev. Lett, 84, 1491 (2000).

[77] J. Knap and M. Ortiz, "Effect of Indenter-Radius Size on Au(001) Nanoindentation," Phys. Rev. Lett, 90, 226102 (2003).

[78] E. B. Tadmor, R. Miller, R. Phillips and M. Ortiz, "Nanoindentation and incipient plasticity," J. Mater. Res. 14, $2233(1999)$. 